\title{
Ernährung bei entzündlichen Darmerkrankungen
}

\author{
Philipp Schreiner $^{\mathrm{a}}$ Maude Martinho-Grueber ${ }^{\mathrm{b}}$ Diana Studerus ${ }^{\mathrm{c}}$ Stephan R. Vavricka ${ }^{\mathrm{a}, \mathrm{d}}$

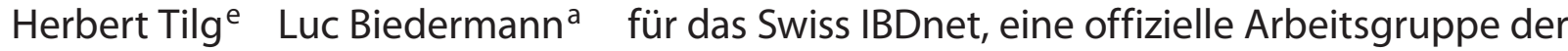 \\ Schweizerischen Gesellschaft für Gastroenterologie
}

${ }^{a}$ Klinik für Gastroenterologie und Hepatologie, Universitätsspital Zürich, Zürich, Schweiz; ${ }^{b}$ Institut für Gastroenterologie, Universitätsklinik für Viszerale Chirurgie und Medizin, Inselspital, Universität Bern, Bern, Schweiz; ${ }^{\top}$ Food on Record ${ }^{\circledR}$ Ernährungsberatung, Basel, Schweiz; ${ }^{d}$ Zentrum für Gastroenterologie und Hepatologie, Zürich, Schweiz; e Universitätsklinik für Innere Medizin I, Gastroenterologie, Hepatologie, Stoffwechsel und Endokrinologie, Medizinische Universität Innsbruck, Innsbruck, Österreich

\section{Schlüsselwörter}

Ernährung · Morbus Crohn · Colitis ulcerosa

\section{Zusammenfassung}

Hintergrund: Die Übernahme einer westlichen Lebensweise, insbesondere der damit verbundenen ernährungsbezogenen Veränderungen, gilt als einer der wichtigsten Faktoren, der zur steigenden Inzidenz entzündlicher Darmerkrankungen (IBD, inflammatory bowel disease), hauptsächlich Morbus Crohn und Colitis ulcerosa, beiträgt.

Zusammenfassung: Die Ernährung stellt für Patienten mit IBD ein zentrales und intuitiv relevantes Thema dar. Auch wenn eine beträchtliche Zahl von Patienten geneigt ist, Ernährungsempfehlungen verschiedenster Anbieter, u.a. aus der Laienpresse, zu befolgen, lassen sich solche Anregungen nur selten durch wissenschaftliche Evidenzdaten untermauern. Dies kann dazu führen, dass Ärzte über verschiedene Aspekte der Ernährung unzureichend informiert sind, was wiederum eine fachgerechte Beratung ihrer Patienten mit IBD von vorne herein ausschließt. Zudem sind Patienten mit IBD gefährdet, Eisen-, Vitamin-B12-, und Folsäuremangel sowie einen Mangel an verschiedenen Mikronährstoffen zu entwickeln, der bei Patienten mit aktiver Erkrankung und solchen, die eine strikte Diät befolgen, besonders stark ausgeprägt sein kann. Ziel der vorliegenden Übersichtsarbeit ist es, die neuesten Daten aus klinischen und epidemiologischen Studien zur Untersuchung der Ernährungsweise und ihrer Auswirkungen auf den Krankheitsverlauf zusammenzufassen und die wichtigsten Nährstoffdefizite bei Patienten mit IBD zu umreißen.

Wichtigste Aussagen: Eine westliche Ernährungsweise mit einem unausgewogenen Verhältnis zwischen den mehrfach ungesättigten Fettsäuren (PUFAs, polyunsaturated fatty acids) Omega- 6 (n-6) und Omega-3 (n-3) zugunsten von n-6-PUFAs kann das Risiko für IBD erhöhen, während eine Ernährungsweise, die reich an Obst und Gemüse ist, das Risiko für IBD verringern kann. Es gibt eine Vielzahl von Ansätzen mit dem Ziel, den Krankheitsverlauf bei IBD durch Ernährungsmaßnahmen zu beeinflussen. Behandlungsstrategien zur Einleitung oder Aufrechterhaltung einer Remission entzündlicher Darmerkrankungen durch eine veränderte Ernährung stecken jedoch noch in den Kinderschuhen, und weitere Anstrengungen in der Ernährungsforschung sind erforderlich, bevor wir solche in der täglichen Praxis anwenden können. Patienten mit IBD, selbst solche in Remission, sind regelmäßig auf Mangelernährung zu untersuchen.

\section{Einführung}

Man geht davon aus, dass die Veränderung von Umweltfaktoren, insbesondere eine Verwestlichung der Lebensweise, verbunden mit einer veränderten Ernährung, verbesserten hygienischen Bedingungen, Impfungen, der zunehmenden Anwendung von Antibiotika [1], der Urbanisierung und einem besseren Zugang zu medizinischer Versorgung, etwa einer vermehrten Anwendung oraler Kontrazeptiva [2-4], einer der Hauptgründe für die steigende Prävalenz entzündlicher Darmerkrankungen (IBD, inflammatory bowel disease) in Ländern mit westlichem Lebensstil ist [5-9]. Den größten Einfluss unter diesen Faktoren auf die Entwicklung von IBD hat wahrscheinlich die Verschiebung hin zu einer westlichen Ernährungsweise, die sich durch einen hohen information@karger.com (c) 2020 S. Karger GmbH, Freiburg www.karger.com/kai

Karger $\stackrel{\text { ! }}{\div}$
Philipp Schreiner

Klinik für Gastroenterologie und Hepatologie

Universitätsspital Zürich

8091 Zürich, Schweiz

Philipp.Schreiner@usz.ch 
Anteil an Proteinen und (ungesättigten) Fettsäuren, aber einen niedrigen Anteil an Gemüse, Ballaststoffen und Obst auszeichnet. Des Weiteren kann infolge dieser «Verwestlichung» der Ernährungsgewohnheiten bei anfälligen Personen durch Veränderungen im Darmmikrobiom und eine gestörte Funktion der Epithelbarriere ein proinflammatorisches Milieu entstehen [10].

Auch wenn keine Leitlinien vorliegen, die eine spezielle Diät während eines Krankheitsschubs befürworten [11-14], nehmen rund $70 \%$ der Patienten mit IBD an, dass ihre Erkrankung durch die Ernährung beeinflusst wird [15], und knapp $60 \%$ von diesen gehen davon aus, dass die Ernährung eine wesentliche Rolle für das Auslösen eines Krankheitsschubs spielt [16]. Auch wenn Patienten mit IBD großes Interesse an dem Thema Ernährung haben, sind lediglich $8-16 \%$ mit den Informationen, die sie von ihren Ärzten erhalten, zufrieden [18]. Interessanterweise konzentrieren sich Patienten in dem Versuch, ihre Ernährungsgewohnheiten zu verändern, eher darauf, bestimmte Nahrungsmittel zu meiden als darauf, ihre Aufnahme von Nahrungsbestandteilen mit wahrscheinlich günstigeren Eigenschaften zu erhöhen [20]. Diese Einstellung ist möglicherweise für Patienten mit IBD wenig hilfreich, sondern führt bei ihnen eher zur eingeschränkten Aufnahme bestimmter Nahrungsmittel mit erheblichen Auswirkungen auf das Sozialleben [17] und zum potenziellen Risiko von Nährstoffdefiziten, u.a. von Eisen, Vitamin B12, Kalzium und Vitamin D [23]. Dieses Vermeidungsmuster bestimmter Nahrungsmittel ist bei Patienten mit strikturierendem Morbus Crohn (MC) besonders stark ausgeprägt [24], was diese Patientengruppe hoch vulnerabel für einen Mangel an Mikronährstoffen macht.

In der vorliegenden Übersichtsarbeit fassen wir den derzeitigen Wissensstand und die aktuellen Daten zum Thema IBD und Ernährung zusammen, beleuchten Nahrungskomponenten und ihr Potenzial, den Verlauf von IBD zu modifizieren, und diskutieren, wie sich Nährstoffdefizite bei Patienten mit IBD am besten erkennen, behandeln und verhindern lassen.

\section{Ernährung und Risiko für IBD}

Die weltweit steigende Inzidenz von IBD [25] wird begleitet von einer «Verwestlichung» der Ernährungsgewohnheiten in Entwicklungsländern. Es liegt daher auf der Hand, dass über genetische Einflüsse hinaus [26] auch Umweltfaktoren, insbesondere die Ernährungsweise, zweifellos eine wesentliche Rolle bei der Entwicklung von IBD spielen [8, 9, 27].

Der exakte Pathomechanismus, wie Ernährung die Entwicklung von IBD begünstigt, ist noch ungeklärt, wobei im Wesentlichen zwei mechanistische Erklärungsmodelle herausstechen, auf die hier eingegangen werden soll.

Zum ersten hat die Ernährung bedeutende Auswirkungen auf die Zusammensetzung der Darmmikrobiota, und Veränderungen der Nahrungszusammensetzung in der frühen Kindheit oder selbst zu einem späteren Zeitpunkt im Leben können eine anti- oder eine proinflammatorische Zusammensetzung der Darmflora begünstigen [28-30]. Die westliche Ernährungsweise zeichnet sich durch eine geringere mikrobielle Vielfalt [31], ein erhöhtes Vorkommen von Bacteroides und Enterobacteriaceae [28-30, 32-34] und einen niedrigeren Anteil an Firmicutes [28] aus. Diese Veränderung der Ernährungsweise in Industrieländern kann zu einer Verschiebung der Mikrobiotazusammensetzung führen und so bei anfälligen Patienten ein proinflammatorisches Milieu begünstigen.

Zum zweiten kann die Ernährung, abgesehen von mikrobiellen Veränderungen, über verschiedene Mechanismen, etwa eine erhöhte Durchlässigkeit der Darmwand, eine verminderte Anzahl von Treg-Zellen im Colon und vermehrte proinflammatorische Marker, auch zu einer veränderten Immunität des Wirts führen [39]. Eine westliche Ernährungsweise mit hohem Fett- und Zuckeranteil reduzierte bei anfälligen Mäusen die Schleimhautdicke, steigerte die Durchlässigkeit der Darmwand und erhöhte die Sekretion von Tumornekrosefaktor a [40].

Es gibt eine Vielzahl möglicher Kandidaten, die eine Entzündungsreaktion im Darm induzieren können. Neben Proteinen, Fetten und Kohlenhydraten können auch Mikropartikel und chemische Stoffe in Nahrungsmitteln wie Emulgatoren oder Verdickungsmittel zu intestinalen und möglichen daraus entstehenden systemischen Entzündungen beitragen.

Interessanterweise können Lebensmittelzusatzstoffe wie Emulgatoren gleichzeitig auf beide oben genannten Mechanismen wirken [41], was ebenfalls unten näher ausgeführt wird.

\section{Fette}

Die mehrfach ungesättigten Fettsäuren (PUFAs) Omega-6 (n-6) und Omega-3 (n-3) zählen zu den am besten untersuchten Nährstoffen, deren Aufnahme in einen potenziellen Zusammenhang mit IBD gebracht wird. n-6-PUFAs (Linolsäure, Arachidonsäure) sind Vorstufen proinflammatorischer Eikosanoide, während n3-PUFAs (Docosahexaenoidsäure (DHA), Eicosapentenoidsäure und alpha-Linolensäure (ALA)) Vorläufer von Eikosanoiden sind, denen entzündungshemmende Eigenschaften zugeschrieben werden [48]. Sonnenblumenöl sowie zahlreiche andere Pflanzenöle und Margarine sind reich an n-6-PUFAs. Ein hoher Gehalt an n-3-PUFAs findet sich hingegen in fettem Fisch und Lebertran (DHA/Eicosapentenoidsäure) sowie in Leinöl und grünem Blattgemüse (ALA). Die veränderten Ernährungsgewohnheiten in westlichen bzw. westlich beeinflussten Ländern zeichnen sich durch einen Anstieg des n- 6/n-3-Quotienten aus [49].

Eine der ersten epidemiologischen Studien zur Untersuchung von Ernährungsfaktoren und der Zunahme an IBD in asiatischen Ländern ist eine japanische Studie. In dieser konnte ein Zusammenhang bzw. eine Korrelation zwischen einem erhöhten n-6/n3-Verhältnis und einer erhöhten Inzidenz von MC nachgewiesen werden [50]. Eine prospektive Kohortenstudie [51] ergab für Patienten mit einer hohen Aufnahme der n-6-PUFA LA ein doppelt so hohes Risiko für die Entwicklung von Colitis ulcerosa (CU). Eine weitere Fall-Kontroll-Studie bei kanadischen Kindern mit MC kam zu ähnlichen Ergebnissen: Kinder mit einem höheren Verhältnis zwischen n-3- und n-6-Fettsäuren hatten ein niedrigeres Risiko für MC [52]. Die protektive Wirkung von n-3-Fettsäuren scheint nicht nur für Kinder zu gelten, sondern auch für Erwachsene über 45 Jahre [53]. In dieser Kohorte war die Gesamt- 
aufnahme von n-3-Fettsäuren über die Nahrung, insbesondere von Docosahexaenoidsäure, negativ mit der Entwicklung von CU assoziiert.

Diese Ergebnisse werden durch die Kohorte der prospektiven «Nurses' Health»-Studie untermauert, in der eine höhere Langzeitaufnahme von n-3-PUFA mit einem niedrigeren Risiko für CU und eine höhere Aufnahme ungesättigter Trans-Fettsäuren mit einem erhöhten Risiko für CU assoziiert war [54].

Eine neuere Metaanalyse zu diesem Thema lässt allerdings darauf schließen, dass zwischen Fettaufnahme und CU-Risiko eher kein Zusammenhang besteht [55]. Andererseits - und im Gegensatz zu zahlreichen anderen Studien, in denen n-3-PUFA eine protektive Wirkung im Hinblick auf das Risiko für IBD zugeschrieben wird - wurde in einer kleinen japanischen Fall-Kontroll-Studie eine positive Assoziation zwischen n-3-PUFA und dem Risiko für MC ermittelt. Die Autoren argumentieren, der Verzehr von fettem Fisch mit einem hohen Gehalt an n-3-PUFA erhöhe zwangsläufig auch die Fettaufnahme insgesamt und steigere somit möglicherweise das Risiko für IBD [56].

Zusammengefasst ist die aktuelle Datenlage eher widersprüchlich. Die Mehrzahl der Studien lässt bei einer höheren Aufnahme von n-6-PUFA (bzw. einem höheren n-6/n-3-Quotienten) auf ein steigendes Risiko für IBD sowie auf eine mögliche protektive Wirkung von n-3-PUFA schließen [54, 57].

\section{Proteine}

Nahrungsproteine stammen aus einer Vielzahl verschiedener Lebensmittel und lassen sich in pflanzliche und tierische Proteinquellen einteilen. In Anbetracht der Rolle, die Proteine in der Ernährung spielen, ist eine Unterscheidung zwischen tierischen und pflanzlichen Proteinen ganz offensichtlich notwendig, da Proteine tierischer Herkunft häufig mehr gesättigte Fette enthalten als pflanzliche Proteine. In der epidemiologischen Analyse von Shoda et al. [50] war eine hohe Zufuhr von tierischem Eiweiß der stärkste unabhängige Risikofaktor für eine erhöhte Inzidenz von MC. Diese Ergebnisse werden durch eine vor kurzem veröffentlichte Prospektivstudie (die E3N-Prospektivstudie), an der französische Frauen mittleren Alters teilnahmen, bestätigt [59]. Ebenso wie in der japanischen Studie zeigte sich die positive Assoziation zwischen Proteinaufnahme und IBD-Risiko lediglich für tierische, nicht jedoch für pflanzliche Proteine.

Laut einer Metaanalyse erhöhte der Verzehr von Fleisch, einem Lebensmittel reich an tierischem Eiweiß, das Risiko für IBD nachweislich, aber aufgrund von methodischen Fehlern in den berücksichtigten Studien ist der Zusammenhang nicht eindeutig [61]. Interessanterweise konnte der Zusammenhang mit einem höheren Risiko für IBD auch bei einem hohen Konsum von Fisch, einer weiteren Quelle von tierischem Eiweiß, nachgewiesen werden $[59,60]$.

\section{Kohlenhydrate}

Auch wenn die Datenlage nicht eindeutig ist, zeigen die meisten Studien doch eine positive Assoziation zwischen einer hohen Aufnahme von raffiniertem Zucker und der Entwicklung von
MC [56, 62], nicht aber der Entwicklung von CU [56, 60]. Allerdings ist bei bestehender CU eine hohe Zufuhr von Saccharose, einer zu den Kohlenhydraten zählenden Zuckerart, möglicherweise mit einem höheren Risiko verbunden [63]. Ungeachtet kürzlich veröffentlichter Daten $[64,65]$, die für Patienten mit einem Ernährungsmuster reich an Zucker und gesüßten Getränken ein erhöhtes Risiko für CU zeigen, konnten diese Ergebnisse durch Daten zweier großer prospektiver Kohortenstudien aus Schweden nicht bestätigt werden [66]. Diese Studien erbrachten keinen Nachweis für einen Zusammenhang zwischen IBD und gesüßten Getränken, wobei hier zwischen verschiedenen künstlichen Süßstoffen nicht unterschieden wurde. Somit besteht weiterhin die Möglichkeit, dass bestimmte künstliche Süßstoffe das Risiko für IBD begünstigen könnten.

Im Gegensatz zu der möglichen negativen Wirkung von Zuckern im Allgemeinen können Obst und Gemüse, die ebenfalls reich an Kohlenhydraten sind, das Risiko für MC, nicht jedoch das Risiko für CU verringern [52, 60, 67]. In zahlreichen Studien wurden gezielt Ballaststoffe untersucht, die sich in großen Mengen nicht nur in Obst und Gemüse, sondern auch in Vollkornprodukten und Hülsenfrüchten finden. In einer großen Prospektivstudie senkte die Langzeitzufuhr von Ballaststoffen, insbesondere aus Obst und zu einem geringeren Teil aus Gemüse, das Risiko für MC, nicht jedoch das Risiko für CU. In der Gruppe mit der höchsten Aufnahme (24,3 g/Tag) war das Risiko für MC um 40\% niedriger [68]. In einer Metaanalyse konnte eine signifikante Dosis-WirkungsBeziehung zwischen Ballaststoffzufuhr und MC-Risiko beobachtet werden, wobei das Risiko bei jeder Steigerung der Ballaststoffzufuhr um $10 \mathrm{~g} /$ Tag um jeweils 13\% sank [69]. Interessanterweise wirkte sich die Aufnahme von Ballaststoffen aus Vollkornprodukten oder Hülsenfrüchten nicht auf das Risiko für IBD aus [68]. Eine weitere populationsbasierte prospektive Kohortenstudie in Europa (EPIC-IBD) [71] stützt die negative Assoziation zwischen Ballaststoffaufnahme und CU. Im Gegensatz zu den US-Daten von Ananthakrishnan et al. [68], die für eine höhere Ballaststoffaufnahme ein vermindertes Risiko für MC zeigten, konnte in der EPIC-IBD-Studie kein Zusammenhang festgestellt werden. Dabei ist jedoch darauf hinzuweisen, dass unter Umständen auch andere in Gemüse oder Obst vorhandene Nährstoffe für die Senkung des IBD-Risikos verantwortlich sein könnten [63].

Zusammengefasst ist die Datenlage zum Thema Makronährstoffe und IBD-Risiko äußerst uneindeutig. Es lässt sich aber unterstreichen, dass eine westliche Ernährungsweise mit einem höheren Risiko für IBD verbunden ist $[60,76]$ und dass insbesondere eine Ernährung mit einem hohen n-3/n-6-Fettsäuren-Verhältnis, die reich an Obst und Gemüse ist, das Risiko für IBD senkt. Diese Aussagen werden durch die aktuellen Leitlinien der European Society for Clinical Nutrition and Metabolism (ESPEN) gestützt [11].

\section{Ernährung und Krankheitsverlauf}

Auch wenn der Zusammenhang zwischen Ernährung und Entwicklung von IBD aus epidemiologischer und pathophysiologi-
98

Kompass Autoimmun 2020;2:96-103 DOI: $10.1159 / 000509482$ 
scher Sicht von großem Interesse ist, sind Patienten, bei denen eine IBD diagnostiziert wurde, an Empfehlungen in Bezug auf Ernährungsumstellungen mit positiven Auswirkungen auf ihren Krankheitsverlauf interessiert. Aufgrund fehlender belastbarer Belege finden sich weder in den Leitlinien der European Crohn's and Colitis Organisation (ECCO), noch in denen der American Gastroenterological Association (AGA) oder der ESPEN Empfehlungen bezüglich einer konkreten Diät während einer Remission oder aktiven Krankheitsphase. In einer kürzlich veröffentlichten Cochrane-Analyse kamen die Autoren zu dem Schluss, dass eine Wirkung jeglicher Ernährungsmaßnahmen auf MC oder CU nicht gesichert ist [77]. Daher geben Ärzte und Ärztinnen nur ungern konkrete Empfehlungen. Folglich konsultiert die Mehrzahl der Patienten weiterhin eine Vielzahl verschiedener Quellen zwecks Ernährungsempfehlungen, u.a. das Internet [78], deren Beratungsangebot in Bezug auf die Qualität und die Evidenzgrundlage wohl kaum derjenigen einer ärztlichen Beratung überlegen sein dürfte.

Was eine Ernährungsumstellung betrifft, können Patienten entweder bestimmte Nahrungsmittel weglassen (Eliminations- oder Ausschlussdiät) oder ihre Ernährung um spezielle Nährstoffe ergänzen. Hierbei ist es von entscheidender Bedeutung, zwischen Diäten, die eine Remission induzieren oder erhalten und somit den Krankheitsverlauf beeinflussen können, sowie Diäten, die lediglich die Symptome von Patienten mit IBD lindern können, zu unterscheiden.

\section{Eliminations-/Ausschlussdiäten}

Die ausschließliche enterale Ernährung (exclusive enteral nutrition, EEN) ist die einzige wirklich bewährte Form der Diät bei IBD-Patienten, insbesondere bei pädiatrischen Patienten mit MC [81, 82]. Die enterale Ernährung, unterteilt in eine elementare (auf der Basis von Aminosäuren, Zuckern, Fetten, Vitaminen und Mineralstoffen) und eine nicht elementare Diätform (zusammengesetzt aus Oligopeptiden oder ganzen Proteinen), ist eine Flüssigdiät, die oral oder per Sonde verabreicht wird. Aufgrund von Remissionsraten von bis zu $80 \%$ bei pädiatrischen $\mathrm{Pa}$ tienten mit MC [83-85] nach 6- bis 8-wöchiger EEN, einer besseren Wirksamkeit und höheren mukosalen Heilungsrate als unter Kortikosteroiden [86] gilt die Ausschlussdiät als primäre Behandlungsoption bei Kindern mit aktivem luminalem MC [87]. Unabhängig davon, ob es sich um eine elementare oder nicht elementare Diätform handelt, lassen sich vergleichbare Remissionsraten erzielen [81]. Bei erwachsenen Patienten mit MC ist die EEN weniger wirksam zur Einleitung einer Remission als die Gabe von Kortikosteroiden [81]. Des Weiteren stellt ihre geringe Schmackhaftigkeit den Hauptgrund für die extrem seltene Anwendung bei erwachsenen Patienten dar, und die ECCO empfiehlt die EEN nicht als Therapie zur Einleitung einer Remission bei erwachsenen Patienten mit MC [13]. Die Datenlage für eine Anwendung der EEN als Erhaltungstherapie bei Erwachsenen oder Kindern mit inaktivem MC ist unzureichend [91]. Zudem liegt keine Indikation für die Behandlung von CU-Patienten mittels EEN vor.
Die Morbus-Crohn-Ausschlussdiät (Crohn's Disease Exclusion Diet, CDED) besteht in einer reduzierten Aufnahme tierischer und gesättigter Fette, glutenhaltiger Getreide und Emulgatoren sowie in einer gesteigerten Zufuhr von Obst, Gemüse und resistenter Stärke. 50\% der Nahrung werden als PEN (partielle enterale Ernährung) gegeben. Der wichtigste Mechanismus besteht in einer Reduzierung der Nahrungsbestandteile, wobei die zulässigen Nährstoffe möglichen Nährstoffdefiziten entgegenwirken und eine Dysbiose verbessern sollen. Die erste Studie zur Untersuchung der CDED bestätigte ihre Wirksamkeit bei der Einleitung einer Remission bei Kindern mit MC [93]. Eine weitere Studie belegte die Einleitung einer Remission selbst bei Kindern, bei denen eine biologische Therapie versagt hatte [94]. Die Daten der ersten randomisierten kontrollierten Studie zum Vergleich einer CDED plus PEN mit einer EEN über 12 Wochen bei Kindern mit MC ergaben für Woche 6 vergleichbare Ansprech- und Remissionsraten und eine nachhaltige Remission in Woche 12 [95]. Aufgrund des einfacheren Behandlungsansatzes bei gleich hoher Wirksamkeit könnte die CDED in Zukunft zu einer Veränderung der Ernährungsempfehlungen führen. Eine Studie zur Anwendung der CDED bei erwachsenen Patienten mit MC läuft aktuell noch [96].

Bei einer neuartigen Diät mit dem Namen CD-TREAT versucht man, die EEN mit herkömmlichen Nahrungsmitteln, basierend auf der Zusammensetzung der EEN, nachzuahmen [97]. Bei dieser Diätform werden spezifische Komponenten wie Gluten und Laktose ausgeschlossen, und der Ballaststoffanteil ist gering. Der Vorteil der CD-TREAT-Diät gegenüber der EEN ist ihre Schmackhaftigkeit - der limitierende Faktor der EEN bei Erwachsenen. In der Studie erwies sich CD-TREAT bei gesunden Probanden als besser verträglich und induzierte eine ähnliche Wirkung auf das Stuhlmikrobiom wie die EEN. Nach 8-wöchiger Anwendung der CD-TREAT-Diät zeigten 80\% (4/5) ein klinisches Ansprechen, und 60\% (3/5) der pädiatrischen Patienten mit MC befanden sich in Remission. Dieser neuartige Ansatz zur Konzipierung einer Diät basierend auf der bewährten EEN ist interessant und besitzt das Potenzial, weitere Ernährungsinterventionen zu beeinflussen.

Die spezifische Kohlenhydratdiät (specific carbohydrate diet, SCD) basiert auf einem Ausschluss komplexer Kohlenhydrate und verarbeiteter Lebensmittel. Monosaccharide, Hühnchen, Fisch und Hartkäse sind zulässig. Diese Diätform wurde lediglich in Fallreihen und kleinen Retrospektivstudien untersucht. Bei pädiatrischen Patienten mit MC konnten eine symptomatische Verbesserung [98] sowie eine mukosale Besserung nachgewiesen werden [99]. Eine andere, unkontrollierte Studie ergab eine mangelhafte mukosale Heilung unter SCD bei asymptomatischen Kindern mit MC [100]. Kürzlich zeigten sich in einer kleinen Prospektivstudie mit 12 pädiatrischen MC-Patienten eine Verbesserung der klinischen Parameter und Laborwerte sowie Veränderungen im Stuhlmikrobiom [101]. Bei erwachsenen Patienten konnte im Rahmen einer Online-Befragung [102] und einer Fallreihe mit Patienten in Remission unter SCD [103] ein klinischer Nutzen gezeigt werden. Bei Erwachsenen wurden keine Studien 
zur Untersuchung einer Verbesserung der Laborwerte oder der Mukosa durchgeführt.

Man geht davon aus, dass eine vegetarische Ernährung das Entzündungsgeschehen vermutlich herunterreguliert, während die westliche Ernährungsweise proinflammatorisch wirkt und zu einer Dysbiose führt [104]. Rund 4\% der Patienten mit IBD leben vegetarisch [19, 105], 17\% befolgen Ernährungsrestriktionen in der Überzeugung, dass sich dies günstig auf ihre IBD auswirkt [105]. Im Rahmen einer prospektiven Kohortenstudie zu Ernährungsfaktoren bei Patienten mit CU in Remission konnte nachgewiesen werden, dass ein vermehrter Verzehr von Fleisch, insbesondere von rotem Fleisch, das Risiko für einen CU-Schub erhöhte [106]. In einer japanischen Studie mit 22 MC-Patienten in Remission war nach 2 Jahren der Anteil der Patienten, bei denen eine Remission aufrechterhalten werden konnte, in der Gruppe mit semi-vegetarischer Ernährung höher (94\%) als in der Gruppe der Allesesser (33\%) [107]. Eine Querschnittsanalyse bei schweizerischen Patienten mit IBD ergab hingegen keinen Zusammenhang zwischen einer vegetarischen Ernährung und dem Krankheitsverlauf von IBD [105]. In einer kürzlich publizierten randomisierten Prospektivstudie (Food and Crohn's Exacerbation Study) [108] senkte ein geringerer Konsum von rotem Fleisch und verarbeitetem Fleisch bei Patienten in Remission nicht das Risiko für ein symptomatisches Wiederaufflammen von MC.

Neben weniger Proteinen oder Fett nehmen Vegetarier normalerweise mehr Gemüse und Ballaststoffe zu sich als Allesesser. In einem Mausmodell induzierten Nahrungsfette bei Interleukin10-defizienten Mäusen Colitis. Die Autoren folgerten daraus, dass bestimmte Fette bei Wirten mit entsprechender genetischer Veranlagung möglicherweise IBD begünstigen können [109]. Eine kürzlich publizierte Prospektivstudie mit CU-Patienten ergab ein dreimal höheres Risiko für ein Wiederaufflammen der Krankheit bei Patienten, die bestimmte Fettsäuren wie Myristinsäure (enthalten in Palm- und Kokosnussöl sowie in Milchfett) konsumierten, sowie bei Patienten, die ALA zu sich nahmen [110]. Dieses Ergebnis mag überraschend erscheinen, da ALA zur Gruppe der n-3-PUFA zählt, denen eine entzündungshemmende Wirkung zugeschrieben wird [48]. Es liegt allerdings nur eine einzige Studie vor, die eine positive Wirkung von n-3 PUFA auf den Krankheitsverlauf bei IBD zeigt. Bei Patienten mit MC reduzierte ein Fischölpräparat mit einem hohen Gehalt an n-3-PUFA das Risiko für einen Schub im Vergleich mit Patienten, die Placebo-Kapseln erhielten, signifikant um 33\% absolut (28 vs. $69 \%$, $p$ $<0,001)$ [111]. Die Ergebnisse dieser Studie wurden jedoch aufgrund der kleinen Stichprobengröße (78 Patienten) in Frage gestellt. In einer größeren Multicenterstudie (EPIC-1 und EPIC-2), an der > 1000 Patienten mit MC teilnahmen, reduzierten n3-PUFA über einen Beobachtungszeitraum von 1 Jahr das Risiko für ein Wiederaufflammen der Krankheit nicht [112]. Eine kürzlich durchgeführte Metaanalyse gelangte zu denselben Ergebnissen wie die EPIC-Studien, nämlich dass n-3-PUFAs bei IBD, ungeachtet ihrer entzündungshemmenden Wirkung im Darm [113], keinen Effekt auf die Aufrechterhaltung einer Remission haben [114].
Eine Studie von Llewellyn et al. [115] untersuchte über 30 verschiedene Ernährungsformen bei Mäusen. Die Autoren konnten nachweisen, dass eine Diät mit hohem Proteingehalt zur Exazerbation einer Colitis bei Mäusen führte und dass Flohsamen (Psyllium), ein bestimmter Ballaststoff, die Colitis besserte, mit einer erhöhten bakteriellen Vielfalt assoziiert war und die Permeabilität der Darmwand reduzierte. Die Autoren stellen die These auf, dass eine proteinarme Diät mit einem hohen Anteil an ausgewählten günstigen Ballaststoffen bei leichten bis mittelschweren IBD die intestinale Barrierefunktion verbessern und die mikrobielle Belastung verringern könne.

Allerdings konnte in einer systematischen Übersichtsarbeit von Wedlake et al. [117] für die Ballaststoffzufuhr bei Patienten mit MC kein Effekt im Sinne der Einleitung einer Remission oder der Verhütung eines Wiederaufflammens der Erkrankung nachgewiesen werden. Interessanterweise ergab eine vor kurzem publizierte Studie zur Untersuchung eines möglichen Zusammenhangs zwischen dem Risiko für eine Pouchitis und dem Verzehr von Obst [119], einer ballaststoffreichen Nährstoffquelle, bei CUPatienten, die viel Obst zu sich nahmen, eine niedrigere Pouchitis-Rate. Des Weiteren wurde bei Patienten, die viel Obst verzehrten, eine höhere mikrobielle Vielfalt festgestellt.

Die Paläo-Diät, auch als «Steinzeitdiät» bezeichnet, basiert auf dem Konzept, dass die Ernährung unserer Vorfahren natürlicher und somit gesünder war. Sie besteht aus Gemüse, Früchten, Nüssen, Samen, kleinen Fischen/Schalentieren, magerem Fleisch von Kleinwild und verzichtet auf verarbeitete Lebensmittel [120]. Eine Weiterentwicklung der Paläo-Diät ist die AutoimmunprotokollDiät bei IBD. Diese Ernährungsweise wurde in einer offenen unkontrollierten Studie untersucht. Eine Verbesserung der Entzündungsmarker zeigte sich zwar nicht, aber bei allen 11 Patienten wurden eine Symptomlinderung und Aufrechterhaltung der Remission verzeichnet [121].

Auch wenn eine mediterrane Ernährungsweise bekanntlich günstige Auswirkungen auf eine Vielzahl chronischer Erkrankungen hat $[123,124]$ und bei Patienten mit MC antiinflammatorisch sowie [125] Mikrobiota-modifizierend wirkt [126], wurde die Mittelmeerdiät bei IBD noch in keiner randomisierten Studie systematisch untersucht. Eine randomisierte Studie bei Patienten mit CU ist jedoch derzeit im Gange [127].

Gärungsfähige Oligo-, Di- und Monosaccharide sowie Polyole (FODMAPs) sind unzureichend resorbierte kurzkettige Kohlenhydrate, die Blähungen, Abdominalschmerz, Flatulenz und Diarrhoe auslösen können. RDS (Reizdarmsyndrom)-ähnliche Symptome treten bei IBD häufig auf und betreffen rund 39\% der Patienten. Sie sind häufiger bei MC als bei CU [128], und eine Abgrenzung dieser Symptome von entzündungsbedingten Schmerzen kann mühsam und schwierig sein.

Eine geringere Zufuhr von FODMAPs führte bei etwa der Hälfte der IBD-Patienten zu einer Verbesserung von Abdominalschmerzen, Flatulenz und Diarrhoe [129]. Des Weiteren ergab eine randomisierte, placebokontrollierte Doppelblindstudie bei Patienten mit IBD und RDS, die FODMAPs konsumierten, ein höheres Maß an Schmerzen, Blähungen, Flatulenz und Stuhl-
100

Kompass Autoimmun 2020;2:96-103 DOI: $10.1159 / 000509482$ 
drang [130]. Hierbei ist es wichtig zu erwähnen, dass eine Ernährungsweise mit einem niedrigen Anteil an FODMAPs die Symptomatik bei IBD-Patienten verbessern kann, nicht jedoch die Entzündungsaktivität beeinflusst.

Interessanterweise ernähren sich ca. $5 \%[105,131,132]$ der IBDPatienten ohne Zöliakie glutenfrei - das sind mehr als doppelt so viele wie in der Allgemeinbevölkerung [132]. Knapp die Hälfte der Patienten ist der Überzeugung, dass sich diese Ernährungsform günstig auf ihre Erkrankung auswirkt [105], obwohl keine Studien vorliegen, in denen - abgesehen von einer klinischen Besserung - eine positiver Einfluss auf das Entzündungsgeschehen nachgewiesen wurde [131]. Ferner ist die Besserung der abdominellen Symptomatik bei nicht zöliakaler Glutenempfindlichkeit wahrscheinlich eher auf die Vermeidung von Fructanen als von Gluten zurückzuführen [133], somit scheint sich eine FODMAParme Diät stärker auf die Symptomatik auszuwirken als eine glutenfreie Diät.

Das Thema wird zusätzlich noch dadurch verkompliziert, dass bei vielen Diäten Emulgatoren zum Einsatz kommen, die das Darmmikrobiom verändern und eine Colitis begünstigen können [41, 134]. Dieser Effekt scheint unmittelbar mit den Mikrobiota-modulierenden Eigenschaften einiger Emulgatoren zusammenzuhängen, da bei nicht exponierten Mäusen, die einer Stuhlmikrobiota-Transplantation mit Stuhl von zuvor exponierten Mäusen unterzogen wurden, Veränderungen der Schleimhautbarriere induziert werden konnten. Einen weiteren Lebensmittelzusatzstoff stellen in pharmazeutischen Produkten enthaltene TitandioxidNanopartikel dar. Diese begünstigen bei Mäusen mit Dextransulfat-Natrium-Colitis nachweislich intestinale Entzündungen [135]. Außerdem fördert Splenda, ein künstliches Süßungsmittel bestehend aus Sucralose und Maltodextrin, bei MC-suszeptiblen Mäusen eine Veränderung des Mikrobioms mit einer Überbesiedlung durch E. coli und einer Zunahme von Proteobacteria, was in der Folge zu einer erhöhten Myeloperoxidase-Aktivität sowie verstärkter Entzündung und intestinalen Schädigungen führt [136]. Zusammengefasst gibt es mehrere verschiedene Ernährungskonzepte, um auf die Symptomatik von IBD-Patienten einzuwirken, sowie Diäten, die den Krankheitsverlauf von IBD beeinflussen können. Patienten, die sich für eine spezielle Diät interessieren, sollten sich jedoch aufgrund des Risikos einer Fehlernährung oder eines Nährstoffmangels von einem Spezialisten für IBD oder einem Ernährungsexperten beraten lassen.

\section{Nahrungsergänzungsmittel}

In dem thematischen Überblick der ECCO über komplementärmedizinische Verfahren und Psychotherapie bei IBD [137] kommen die Autoren zu dem Schluss, dass keine hinreichenden Belege vorliegen, die eine Anwendung von Vitaminen zur Einleitung oder Aufrechterhaltung einer Remission bei MC und CU stützen. Dessen ungeachtet wurde in einer prospektiven Kohortenstudie nachgewiesen, dass bei CU-Patienten mit einem 25(OH)D-Spiegel unter $35 \mathrm{ng} / \mathrm{ml}$ das Risiko für einen klinischen Schub erhöht ist (Odds Ratio (OR) 1,25, 95 \%-Konfidenzintervall (KI) 1,01$1,56, p=0,044)[138]$.
Bei Patienten mit MC ist der 25(OH)D-Spiegel umgekehrt proportional zur Krankheitsaktivität [139], und bei Patienten mit CU ist der 25(OH)D-Spiegel umgekehrt proportional zur mukosalen Entzündung [140]. Darüber hinaus wiesen Patienten mit niedrigem 25(OH)D-Spiegel im Rahmen einer Längsschnittstudie eine höhere Morbidität und Krankheitsschwere auf [141].

Eine orale Supplementierung mit 1200 IE Vitamin D3 verringerte außerdem bei Patienten mit MC das Risiko für ein Wiederaufflammen der Erkrankung nicht signifikant von 29 auf 13\% ( $p=$ 0,06) [142].

Auch wenn die Daten nicht besonders belastbar sind, ist eine Supplementierung von Vitamin D unbedenklich und gut verträglich, sodass es allen IBD-Patienten mit Vitamin-D-Mangel verabreicht werden sollte [145]. Anthocyan, das in großen Mengen in Heidelbeeren und Oregon-Himbeeren vorkommt, besitzt antiinflammatorische und antioxidative Wirkungen [146]. Eine offene Pilotstudie belegte, dass bei leichter bis mittelschwerer CU ein täglich verabreichtes standardisiertes Anthocyan-reiches Blaubeerpräparat nach 6 Wochen bei 63,7\% der Patienten zu einer Remission und bei 90,9\% zu einem Ansprechen führte.

Ein weiteres pflanzliches Präparat bestehend aus Myrrhe, Kamillenblütenextrakt und Coffeae carbo (Kaffeekohle) wurde in einer randomisierten Doppelblindstudie bei Patienten mit inaktiver CU im Vergleich mit Mesalamin untersucht [152]. Die Rezidivraten nach 12 Monaten unterschieden sich zwischen beiden Gruppen nicht, was die Deutsche Gesellschaft für Gastroenterologie, Verdauungs- und Stoffwechselkrankheiten (DGVS), veranlasste, die Anwendung des Präparats als komplementäre Behandlung im Rahmen einer Erhaltungstherapie bei CU zu empfehlen [153]. Curcumin, ein aus dem indischen Gewürz Kurkuma (Curcuma longa) gewonnenes Polyphenol, wird in der traditionellen chinesischen Medizin und in der ayurvedischen Medizin aufgrund seiner entzündungshemmenden und antioxidativen Eigenschaften verwendet $[154,155]$. In einer randomisierten, placebokontrollierten Doppelblindstudie induzierte Curcumin in einer Dosis von $3 \mathrm{~g}$ täglich bei Patienten, die trotz Behandlung mit der Höchstdosis Mesalamin an leichter bis mittelschwerer aktiver CU litten, wirksamer eine klinische Remission und Response als Placebo [156]. Neben seiner Wirkung in Bezug auf die Einleitung einer Remission ist Curcumin nachweislich, selbst in einer niedrigeren Dosis von $2 \mathrm{~g}$ täglich, in der Lage, bei Patienten mit inaktiver CU eine Remission aufrecht zu erhalten [158]. In dieser randomisierten, placebokontrollierten Doppelblindstudie trat während der 6-monatigen Behandlung bei nur 4,7\% der Patienten, die Curcumin erhielten, ein erneuter Krankheitsschub auf, verglichen mit $20,5 \%$ in der Placebogruppe $(p=0,04)$. Es wurden keine schwerwiegenden Nebenwirkungen verzeichnet. Eine Cochrane-Analyse stützt das Ergebnis, dass Curcumin bei Patienten mit CU eine Remission wirksam aufrechterhält [159].

In Bezug auf die tägliche Anwendung von Curcumin werden zwei wichtige Fakten genannt. Zum ersten beträgt die zulässige tägliche Einnahme, die von der Expertenkommission der Food and Agriculture Organization of United Nations (FAO) und World Health Organization (WHO) für Lebensmittelzusatzstoffe 
(JEFCA) beurteilt wurde [160], 0-3 mg/kg Körpergewicht, was weit unterhalb der in den Studien untersuchten Dosierungen liegt. Aufgrund des Umstands, dass Curcumin nicht als Arzneimittel zugelassen und ausschließlich als frei verkäufliches Nahrungsergänzungsmittel erhältlich ist, liegt es zum zweiten häufig nicht in Reinform vor, sondern enthält zahlreiche Zusatzstoffe. Diese häufig erhältlichen Kurkuma-Kapseln enthalten rund 50 mg Curcumin und Piperin und führen zu einer Erhöhung des Serumspiegels von Curcumin [161]. Somit ist es äußerst schwierig, die in den Studien verwendeten hohen Dosierungen zu erreichen.

\section{Fehlernährung}

Patienten mit aktiven IBDs weisen ein erhöhtes Risiko für eine Fehlernährung auf. Die Prävalenz einer Fehlernährung bei IBD liegt zwischen 6 und 16\%, was verglichen mit Personen ohne IBD einem 5-fach erhöhten Risiko für eine Fehlernährung entspricht $[164,16]$. Durch IBD-bedingte chirurgische Eingriffe in der Vorgeschichte verdoppelt sich das Risiko für eine Fehlernährung. Eine fortgesetzte klinische Aktivität und das Meiden bestimmter Nahrungsmittelgruppen während eines Krankheitsschubs sind sogar mit einem vierfach bzw. zehnfach höheren Risiko für eine Fehlernährung assoziiert [16]. Den stärksten Einfluss auf eine Fehlernährung haben somit vermutlich eine bewusste Einschränkung der Nahrungsaufnahme und ein verminderter Appetit während eines Krankheitsschubs.

Die häufigsten Formen eines Mikronährstoffmangels, aufgeführt mit absteigender Häufigkeit, sind Eisenmangel, Vitamin-D-Mangel, Vitamin-B12-Mangel, Zinkmangel und Folsäuremangel. Die Ursachen eines Mikronährstoffmangels sind multifaktoriell und umfassen u.a. eine eingeschränkte Nahrungsaufnahme, Vitaminverluste über den Darm, Malabsorption und unerwünschte Arzneimittelwirkungen [166].

\section{Eisenmangel}

Eisenmangel tritt mit einer Prävalenz von bis zu 70\% auf und ist die häufigste Ursache für eine Anämie bei Patienten mit IBD (Eisenmangel-Anämie (IDA)). Die zweithäufigste Ursache einer Anämie bei IBD-Patienten ist eine Anämie bei chronischer Erkrankung (ACD), die bei IBD-Patienten häufig im Zusammenhang mit Eisenmangel auftritt. Eisenmangel bei IBD-Patienten ist multifaktoriell und ist durch eine verringerte Eisenaufnahme, eine gestörte Eisenresorption im Duodenum und oberen Jejunum, eine verminderte Eisenresorption aufgrund hoher Hepcidinwerte und chronischen Blutverlust aus der entzündeten Darmschleimhaut bedingt [167].

Die verbreitet angewendeten oralen Eisenpräparate bestehen aus Eisensalzen (Eisensulfat, -fumarat und -glukonat), und die Resorption von Nicht-Hämeisen ist schlecht. Über das Duodenum können pro Tag maximal 10-20 mg Eisen resorbiert werden; ca. $90 \%$ werden nicht resorbiert [174]. Daher werden häufig hohe Dosen verabreicht, was zu ROS-vermittelten toxischen Wirkungen von nicht resorbiertem Eisen auf die Darmschleimhaut [175] und Darmdysbiose führen sowie Entzündungen und Diarrhoe ver- stärken kann $[177,178]$. Weitere kleine Studien belegen eine möglicherweise erhöhte klinische Krankheitsaktivität bei IBD-Patienten nach Einnahme von Eisenfumarat [179] sowie ein geringes, aber unserer Auffassung nach relevantes Rezidivrisiko in Höhe von $6 \%$ [180].

Nichtsdestotrotz kann auf eine orale Eisenaufnahme bei IBD-Patienten nicht komplett verzichtet werden, wobei jedoch eine hoch dosierte orale Eisengabe bei IBD-Patienten zu vermeiden sind, was insbesondere für manche Vitamin-Kombinationspräparate gilt. Bei oraler Gabe sollte Eisen alle zwei Tage in niedrigen Einzeldosen verabreicht werden, um die Eisenresorption zu optimieren [183].

Aufgrund der entzündungsbedingt hohen Hepcidin-Werte [184] kann die orale Eisenresorption bei Patienten mit aktiven IBD stark beeinträchtigt oder sogar unmöglich sein. Aus diesem Grunde empfiehlt die ECCO intravenöses Eisen bei Patienten mit klinisch aktiver Krankheit, bei Patienten mit einer Unverträglichkeit von oralem Eisen oder solchen, die Eythropoese-stimulierende Substanzen benötigen, sowie bei Patienten mit Hämoglobinwerten unter $10 \mathrm{~g} / \mathrm{dl}$ (aufgrund eines sehr viel schnelleren Ansprechens auf intravenöses Eisen) [185].

Nach Korrektur eines Eisenmangels sollten Patienten ihre Eisenwerte alle 3 Monate über mindestens 1 Jahr kontrollieren lassen, und bei einem Abfall des Ferritinspiegels auf unter $100 \mu \mathrm{g} / \mathrm{l}$ sollte eine Eisensubstitution erfolgen [185]. Bei Patienten mit aktiver Krankheit sollten die Werte - unabhängig vom Vorliegen einer Anämie - alle 3 Monate, bei Patienten in klinischer Remission alle 12 Monate kontrolliert werden [188].

\section{Vitamin-B12-Mangel}

Die Angaben zur Prävalenz von Vitamin-B12-Mangel liegen, bedingt durch verschiedene diagnostische Verfahren, zwischen 6 und 38\% [189]. Ein echter Vitamin-B12-Mangel bei asymptomatischen Patienten wird definiert als ein Cobalamin-Serumspiegel $<148$ pmol/l (200 ng/l) und ein erhöhter Homocystein- oder Methylmalonsäure-Serumspiegel [190]. Im Duodenum bindet mit der Nahrung aufgenommenes Cobalamin an den Intrinsic Factor, der von den Parietalzellen synthetisiert wird, und kann so im terminalen Ileum resorbiert werden [191]. Da MC den gesamten Gastrointestinaltrakt befallen kann, tritt ein Vitamin-B12-Mangel bei Patienten mit MC sehr viel häufiger auf als bei Patienten mit CU [168]. Hauptrisikofaktor für einen Vitamin-B12-Mangel ist eine Ileumresektion $>30 \mathrm{~cm}$ [189]. Interessanterweise stellt ungeachtet der Tatsache, dass die Cobalamin-Resorption hauptsächlich im Ileum stattfindet, ein ilealer Befall bei MC keinen Risikofaktor für einen Cobalamin-Mangel dar [189].

Normalerweise ist Vitamin-B12-Mangel bei Patienten mit CU etwa ebenso häufig wie in der Allgemeinbevölkerung. Eine Ausnahme bilden CU-Patienten mit einem ileoanalen J-Pouch. Diese können, wahrscheinlich aufgrund einer geringen bakteriellen Überbesiedlung, an Vitamin-B12-Mangel leiden [192]. In Einzelfällen kann es nach langjähriger Backwash-Ileitis zu einem Vitamin-B12-Mangel kommen. Allerdings wurden hierzu noch keine Untersuchungen durchgeführt.
102

Kompass Autoimmun 2020;2:96-103 DOI: $10.1159 / 000509482$ 


\section{Vitamin-D-Mangel}

Bis zu 60\% der IBD-Patienten, insbesondere Patienten mit der Diagnose MC und erhöhter Krankheitsaktivität [72, 193, 194] sowie Schwangere mit IBD, leiden an Vitamin-D-Mangel [195]. Interessanterweise besitzen junge männliche Patienten ein sehr hohes Risiko [141], und MC-Patienten mit isolierter Kolonbeteiligung bzw. CU-Patienten wiesen eine vergleichbare Prävalenz von $25(\mathrm{OH}) \mathrm{D}$-Spiegeln auf wie MC-Patienten mit einem Befall des Ileums und Kolons oder des Dünndarms [141]. Daneben konnte in einer Metaanalyse bei Patienten mit IBD eine höhere Prävalenz für einen Vitamin-D-Mangel nachgewiesen werden als bei Gesunden [196]. Die Ursachen für einen Vitamin-D-Mangel bei IBD sind multifaktoriell. Es gibt 2 natürliche Quellen von Vitamin D, nämlich Ergocalciferol (Vitamin D2), das in Nahrungsmitteln wie insbesondere fettem Fisch und Pilzen enthalten ist, sowie Vitamin D3, das bei UVBStrahlung in der Epidermis gebildet wird [197]. Da Vitamin-DMangel in den meisten nördlichen Ländern in der Allgemeinbevölkerung ein weit verbreitetes Problem darstellt, zählt eine Vitamin-D-Malabsorption vermutlich nicht zu den zentralen Hauptursachen für den Vitamin-D-Mangel bei IBD. Vielmehr zeigt sich bei Patienten mit IBD eine unzureichende Exposition gegenüber Sonnenlicht, und durch die Entzündung werden $\mathrm{Zy}$ tokine hochreguliert, die in der Folge den 25(OH)D-Serumspiegel senken [197].

Auch wenn die Daten bezüglich einer potenziellen günstigen Wirkung auf den Krankheitsverlauf bei IBD nicht eindeutig sind, spielt Vitamin D eine wichtige Rolle für die Knochengesundheit. Insbesondere bei Patienten mit IBD, die vermutlich wiederholt Kortikosteroide benötigen, sind niedrige 25(OH)D-Spiegel zu vermeiden. Da die Therapietreue bei Patienten mit chronischen Erkrankungen ein wichtiger limitierender Faktor ist, stellt eine Vitamin-D-Substitution mit 45000 IE einmal monatlich eine pragmatische Vorgehensweise dar.

\section{Fazit}

Die westliche Lebensweise, insbesondere eine westliche Ernährung, ist eine der Hauptursachen für die erhöhte Prävalenz von IBDs in Industrie- und Schwellenländern. Eine erhöhte Aufnahme von Fetten und/oder Proteinen, der geringere Verzehr von Obst und Gemüse sowie ein vermehrter Einsatz von Emulgatoren oder anderen Bindemitteln gehören zu den wichtigsten potenziellen Faktoren, die Entzündungen im Darm gesunder Personen begünstigen. Bislang ist jedoch unklar, welche dieser einzelnen Ernährungsbestandteile epidemiologisch betrachtet in erster Linie für die aktuelle Zunahme von IBD verantwortlich sind.

Auch wenn zahllose Bücher und Internetseiten zum Thema Ernährung und IBD vorliegen, gibt es nur wenig Belege, die restriktive diätetische Interventionen bei Patienten mit IBD stützen. Daher sollten wir unseren Patienten bei unseren aktuellen Empfehlungen im Wesentlichen zu einer gesunden, ausgewogenen Ernährung auf der Basis nicht verarbeiteter Lebensmittel anstatt zu einer Einschränkung und/oder Supplementierung bestimmter Nahrungskomponenten raten.

\section{Danksagung}

Keine.

\section{Disclosure Statement}

Die Autoren erklären, dass keine Interessenskonflikte bestehen.

\section{Finanzierung}

Es gab keine Finanzierungsquellen.

\section{Beiträge der einzelnen Autoren}

P. S. Konzeption und Studiendesign, Erarbeitung des Artikelentwurfs, Genehmigung der endgültigen Fassung. M. M.-G., D. S., S. R. V. und H. T. Studiendesign, kritische Überprüfung und Genehmigung der endgültigen Fassung. L. B. Konzeption und Studiendesign, kritische Überprüfung und Genehmigung der endgültigen Fassung.

\section{Literatur}

Die Literatur ist unter www.karger.com/Article/Fulltext/509480 abrufbar. 